Method Cross-sectional study that included 72 workers of the Mexico-Puebla Highway cabins (guards); 60 cashiers and 12 workers in charge of the shift, in which socio- demographic, clinical and occupational variables were explored; haemoglobin $(\mathrm{Hb})$, hematocrit (HCT) and carboxyhemoglobin $(\mathrm{COHb})$ was determined in their blood, and it was applied the CONSB for CO. The workers who are in charge of the full shift integrated the less exposed to $\mathrm{CO}$ group, while the cashiers would shape the greater exposure group.

Results In 49 workers, $\mathrm{Hb}: 18.1 \pm 1.9 \mathrm{mg} / \mathrm{dL}$ and Hcto: 55.3 \pm 8.7 without significant differences in the presence or absence of erythrocytosis between exposure groups $(\mathrm{p}=0.82)$. In the neuropsychological tests of 72 workers, greater alteration was identified in the exposed group, only in the test of symbol and digits $(p=0.001)$, trail making $B(p=0.002)$, and test of digits $(p=0.003)$; the test of building with blocks resulted borderline $(p=0.07)$. In all tests except in the test of learning of Rey, the non-exposed showed better performance.

Conclusions Despite what has been achieved, it is important to follow up the erythrocytosis and the neuropsychological effects on all workers, in addition to start treatment to prevent complications. The perception of innocuousness of chronic exposure to $\mathrm{CO}$ in these workersmust avoid.

\section{PREVALENCE OF OBESITY AND RISK OF METABOLIC COMPLICATIONS AMONG WORKERS OF A HARNESS PLANT}

Eloísa Esquivel Rodríquez, Osmel La Llave León, Mónica Gallegos Alvarado, Armida Rodríguez Salazar, Angélica Ma. Lechuga Quiñones. Martha Lilia Parra Domínguez; Universidad Juárez Del Estado de Durango, Durango, Mexico

\subsection{6/oemed-2014-102362.339}

Objectives To explore the prevalence of obesity and risk of metabolic complications among workers of a harness plant in Durango, Mexico.

Method A croos-sectional study was conducted on 300 workers. Measurements of body mass index (BMI) and waist hip ratio (WHR) were obtained. BMI $\left(\mathrm{m} / \mathrm{kg}^{2}\right)$ was classified into underweight $(<18.5)$, normal range (18.5-24.9), pre-obese (25.029.9), obese class I (30.0-34.9), obese class II (35.0-39.9), and obese class III $(\geq 40.0)$. Risk of metabolic complications (RMC) was considered high when WHR $>0.85$ for women, and $>$ 0.95 for men; and moderated between $0.80-0.85$ for women and $0.90-0.95$ for men. Analysis of variance (ANOVA) was applied for continuos variables, and $\chi^{2}$ test for categorical variables.

Results The mean age $( \pm \mathrm{SD})$ of the subjects was $28.7( \pm 8.9)$ yrs. In the sample, $51.7 \%$ were male. According to the BMI, $47.3 \%$ of participants were in the normal range, $35.3 \%$ preobese, and $15.7 \%$ showed obesity. According to the WHR, 30\% were at high risk, and $28.6 \%$ at moderate risk for developing metabolic complications. The RMC (high and moderate) was significant more prevalent in women than in men, $77.5 \%$ vs $49.3 \%$, $(\mathrm{p}<0.0001)$. ANOVA yielded significant variation in obesity according to age $(\mathrm{p}<0.001)$. Post hoc Tukey test showed differences of obese class II with normal range $(p=0.007)$ and underweight $(\mathrm{p}=0.0169)$.

Conclusions The prevalence of obesity and RMC are quite high in studied population. The risk is higher among women and increases with age.

\section{THE SHIFT WORK AND THE BURNOUT SYNDROME}

Patricia Baptista, Renata Tito, Vanda Felli, Fabio Silva, Silmar Silva. University of Sao Paulo, Sao Paulo, Brazil

\subsection{6/oemed-2014-102362.340}

Objectives This study aims to identify the presence of burnout in nursing workers of a intensive care skilled in children with heart disease.

Method This is an exploratory, transversal and quantitative study with 92 nursing workers of a children intensive care in a university hospital of Sao Paulo, skilled on cardiology. The data were collect by MBI - Maslach Burnout Inventory. The data were analysed statistically and were presented in graphs and tables.

Results The results showed that the subjects have on average 31 years old, $93.5 \%$ were female, $69,3 \%$ worked less than 10 years in a paediatric and neonatal cardiology unit, and $79.3 \%$ have only one employment. It was observed that burnout levels were considered high in $8(8,7 \%)$ of the participants and shows that $46(50 \%)$ of workers have a high score for at least one of the three dimensions. The occurrence of burnout among workers in the study reveal the need for studies in the field of nursing paediatric proposed prevention and intervention to subsidise worker health nursing.

Conclusions These data suggest that shift work impacts the quality of life of this population, since living with physical and psychological changes, which may also influence the quality of nursing care. Therefore, it's necessary to recreate the work process with the implementation of protective measures the health of nursing.

\section{MENTAL HEALTH PROBLEMS OF SEAFARERS}

leva Malinauskaite, Lina Bernotaite. Lithuanian University of Health Sciences, Kaunas, Lithuania

\subsection{6/oemed-2014-102362.341}

Objectives Recent investigations have shown that workplace bullying as a long lasting traumatic experience with the symptoms of victimisation may lead to psychological distress. The objective of our study was to investigate the prevalence of workplace bullying among seafarers and the associations with psychological distress in Klaipeda, Lithuania.

Method In this obseravtional study, totally 450 sefarers were investigated (response rate 68\%). We used the anonymous questionnaire with demographical variables, Negative acts questionnaire and GHQ-12. The SPSS 17.0 was used in the statisciacal analysis. Logistic regression analysis was employed for the associations between workplace bullying and mental distress among seafarers, controlling for variety of demographical and pyschosocial factors.

Results The prevalence of occasional bullying among seafarers was $10.1 \%$, severe bullying $3.8 \%$, mental distress $9.3 \%$. The OR of occasional bullying for psychological distress was $2.92 ; 95 \% \mathrm{CI}$ 0.99-8.61, of severe bullying 4.60; 95\% CI 1.13-18.78. After adjustment for nationality, age, occupational position the OR of occasional bullying for psychological distress was 3.47; 95\% CI 0.95-12.63, of severe bullying 6.52 ; 95\% CI 1.34-31.76.

Conclusions The significant associations between workplace bullying and psychological distress implicate that the preventive measures should be directed towards the improvement of the psychological climate in the workplace for health promotion strategies in seafaring. 